\title{
Nucleation and Growth Mechanisms for Pd-Pt Bimetallic Nanodendrites and Their Electrocatalytic Properties
}

\author{
Byungkwon Lim ${ }^{1}$, Majiong Jiang ${ }^{2}$, Taekyung Yu ${ }^{1}$ Pedro H. C. Camargo ${ }^{1}$, and Younan Xia ${ }^{1}(\varangle)$ \\ ${ }^{1}$ Department of Biomedical Engineering, Washington University, St. Louis, Missouri 63130, USA \\ ${ }^{2}$ Department of Chemistry, Washington University, St. Louis, Missouri 63130, USA \\ Received: 14 November 2009 / Revised: 25 November 2009 / Accepted: 27 November 2009 \\ (C) The Author(s) 2010. This article is published with open access at Springerlink.com
}

\begin{abstract}
In a seed-mediated synthesis, nanocrystal growth is often described by assuming the absence of homogeneous nucleation in the solution. Here we provide new insights into the nucleation and growth mechanisms underlying the formation of bimetallic nanodendrites that are characterized by a dense array of Pt branches anchored to a $\mathrm{Pd}$ nanocrystal core. These nanostructures can be easily prepared by a one-step, seeded growth method that involves the reduction of $\mathrm{K}_{2} \mathrm{PtCl}_{4}$ by $\mathrm{L}$-ascorbic acid in the presence of 9-nm truncated octahedral Pd seeds in an aqueous solution. Transmission electron microscopy (TEM) and high-resolution TEM analyses revealed that both homogeneous and heterogeneous nucleation of Pt occurred at the very early stages of the synthesis and the Pt branches grew through oriented attachment of small Pt particles that had been formed via homogeneous nucleation. These new findings contradict the generally accepted mechanism for seeded growth that only involves heterogeneous nucleation and simple growth via atomic addition. We have also investigated the electrocatalytic properties of the $\mathrm{Pd}-\mathrm{Pt}$ nanodendrites for the oxygen reduction and formic acid oxidation reactions by conducting a comparative study with foam-like Pt nanostructures prepared in the absence of Pd seeds under otherwise identical conditions.
\end{abstract}

\section{KEYWORDS}

Palladium, platinum, seeded growth, oxygen reduction, formic acid oxidation

\section{Introduction}

Understanding of nucleation and growth mechanisms is a prerequisite for the rational design of metal nanostructures with desired properties. Recently, heterogeneous, seeded growth has emerged as a powerful tool for precisely controlling the morphology and composition of bimetallic nanostructures [1-13]. This technical approach has enabled the preparation of a wide variety of bimetallic nanostructures that cannot be achieved otherwise. Notable examples include nanocubes [1-3] and nanoplates [4-6] with well-defined core-shell structures, highly anisotropic nanorods consisting of a secondary metal deposited at the tips of pre-formed nanorods [7-9], and nanodendrites characterized by a dense array of branched arms made of one metal supported on a core of another metal [10-13].

In heterogeneous, seeded growth, pre-formed nanocrystals are generally considered to serve as primary sites (i.e., seeds) for the nucleation of another metal with a different chemical identity and nanocrystal

Address correspondence to xia@biomed.wustl.edu 
Nano Res (2010) 3: 69-80

growth is often described by assuming the absence of homogeneous nucleation in the solution. Experimentally, this postulation has been supported by observations that include the absence of isolated particles made of a secondary metal in the final product and/or the epitaxial relation between the core and the shell. A recent theoretical study based on Monte Carlo simulations of heterogeneous nucleation has shown that foreign seeds larger than a critical size can indeed lower the free-energy barrier for a nucleation process and the rate of homogeneous nucleation is vanishingly small [14]. Nevertheless, the exact mechanism of seeding by a foreign nanocrystal still remains largely unresolved due to the lack of evidence for nucleation and growth occurring at early stages of a synthesis.

Recently, Pd-Pt and Au-Pt bimetallic nanodendrites have been synthesized using the seeded growth method by our and other research groups [10-13]. These bimetallic nanodendrites are of particular interest due to their high specific surface areas, and have shown great promise for use as catalysts or electrocatalysts with high activity. In previous studies, however, a mechanism that only considers heterogeneous nucleation and simple growth via atomic addition was suggested to account for the formation of such bimetallic nanostructures without direct evidence for nanocrystal growth in solution. Here we provide new insights into the nucleation and growth mechanisms underlying the formation of a bimetallic nanodendrite that consists of $\mathrm{Pt}$ branches anchored to a $\mathrm{Pd}$ nanocrystal core. In this new work, we discovered that both homogeneous and heterogeneous nucleation of Pt occurred at the very early stages of seeded growth and led to the formation of discrete small Pt particles in the solution and Pt bumps at multiple sites on the $\mathrm{Pd}$ seed, respectively. Furthermore, we also found that the growth of Pt branches mainly proceeded via oriented attachment of the initially formed $\mathrm{Pt}$ particles. The new results presented herein contradict the previously reported mechanisms and provide clear evidence for the involvement of homogeneous nucleation in the heterogeneous, seeded growth of bimetallic nanodendrites. In addition to the mechanistic study, we systematically investigated the electrocatalytic properties of the Pd-Pt nanodendrites by conducting a comparative study with foam-like $\mathrm{Pt}$ nanostructures prepared in the absence of Pd seeds under otherwise identical conditions. We have applied these nanostructures not only as a cathode catalyst for the oxygen reduction reaction (ORR) but also as an anode catalyst for the formic acid oxidation reaction in a proton-exchange membrane (PEM) fuel cell.

The Pd-Pt nanodendrites were synthesized in an aqueous solution by reducing $\mathrm{K}_{2} \mathrm{PtCl}_{4}$ with $L$-ascorbic acid in the presence of 9-nm truncated octahedral $\mathrm{Pd}$ seeds, with the addition of poly(vinyl pyrrolidone) (PVP) as a stabilizer [12]. Transmission electron microscopy (TEM) and high-resolution TEM images of the Pd seeds are shown in Fig. S-1 of the Electronic Supplementary Material (ESM). To investigate the morphological evolution of the Pd-Pt nanodendrites, aliquots of the reaction solution were taken out at various stages and examined using electron microscopy. Figures 1(a)-1(d) show typical TEM images of the samples that were taken out at 1, 2, 5, and $10 \mathrm{~min}$, respectively. For comparison, we also studied the morphological evolution of $\mathrm{Pt}$ nanostructures in the absence of Pd seeds under otherwise identical conditions (Figs. $1(\mathrm{e})-1(\mathrm{~h})$ ). At $t=1 \mathrm{~min}$, the $\mathrm{Pd}-\mathrm{Pt}$ sample contained a large number of small $\mathrm{Pt}$ particles with sizes less than $3 \mathrm{~nm}$ in addition to Pt-decorated Pd seeds (Fig. 1(a)), indicating that both homogeneous and heterogeneous nucleation of $\mathrm{Pt}$ occurred at the early stages of the reaction. Figure 2(a) gives a highresolution TEM image of a single $\mathrm{Pd}-\mathrm{Pt}$ particle, clearly showing the formation of $\mathrm{Pt}$ bumps via heterogeneous nucleation at multiple sites on the truncated octahedral Pd seed. The small Pt particles formed via homogeneous nucleation had a singlecrystal structure, as revealed by the high-resolution TEM image in Fig. 2(b). As the reaction proceeded to $t=5 \mathrm{~min}$, the number of small $\mathrm{Pt}$ particles rapidly decreased while a number of $\mathrm{Pt}$ branches started to appear on each $\mathrm{Pd}$ seed in random directions (Figs. 1(b) and 1(c)). These observations indicate that the $\mathrm{Pt}$ branches were formed through attachment of the initially formed, small Pt particles. Some secondary branches also began to grow from the main branches during this period. In the following $5 \mathrm{~min}$, essentially all the small $\mathrm{Pt}$ particles disappeared and the $\mathrm{Pd}-\mathrm{Pt}$ 
particles evolved into a highly branched structure with an overall dendritic morphology (Fig. 1(d)). The $\mathrm{Pt}$ branches were distributed over the entire surface of the Pd core and did not overlap appreciably. Growth via particle attachment was also observed in the absence of Pd seeds (Figs. 1(e)1(h)). In this case, however, the small Pt particles that had been formed via homogenous nucleation self-aggregated into a denser nanostructure with a foamlike morphology. Both the nanostructures, i.e., $\mathrm{Pd}-\mathrm{Pt}$ nanodendrites and foam-like $\mathrm{Pt}$ aggregates, did not exhibit any morphological change during the next $3 \mathrm{~h}$ (Fig. S-2 in the ESM).

We suggest that in the synthesis of the Pd-Pt nanodendrites, homogeneous nucleation of the $\mathrm{Pt}$ occurred as follows. In the initial stage of synthesis, the degree of supersaturation for $\mathrm{Pt}$ atoms increased rapidly due to the fast reduction of $\mathrm{K}_{2} \mathrm{PtCl}_{4}$ precursor by $L$-ascorbic acid. However, the number of possible nucleation sites provided by the Pd seeds might not be sufficient to accommodate all the $\mathrm{Pt}$ atoms generated from the reduction and, as a result, the supersaturation would be kept at a high level, facilitating homogeneous nucleation in the solution $[15,16]$. Accordingly, the Pt atoms that could not nucleate on the surfaces of $\mathrm{Pd}$ seeds would self-nucleate to form small Pt particles.

The observed attachment of $\mathrm{Pt}$ particles might be related to the ineffective stabilization by PVP. It has been shown that PVP is less effective as a stabilizer in a polar medium such as water [17-19]. The small particles have a higher chemical potential due to a large surface area-to-volume ratio, and particle attachment can reduce surface energy by reducing the surface area. We attribute the formation of $\mathrm{Pt}$ branches to the oriented attachment mechanism
[20-27], where adjacent particles self-organize so that they share a common crystallographic orientation. Figure 2(c) shows a high-resolution TEM image of a single $\mathrm{Pd}-\mathrm{Pt}$ nanodendrite. The lattice fringes were

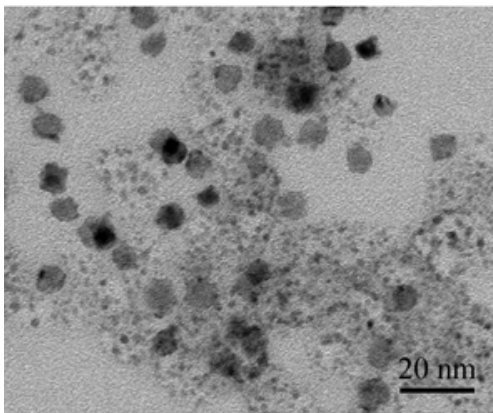

(a)

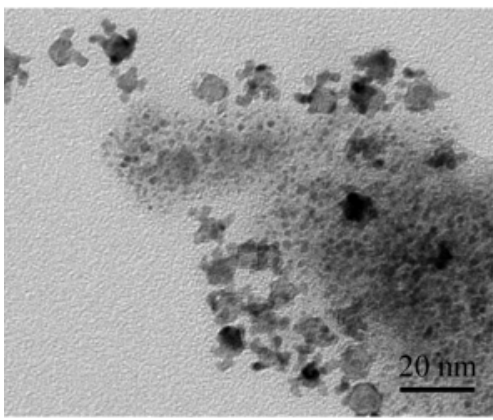

(b)

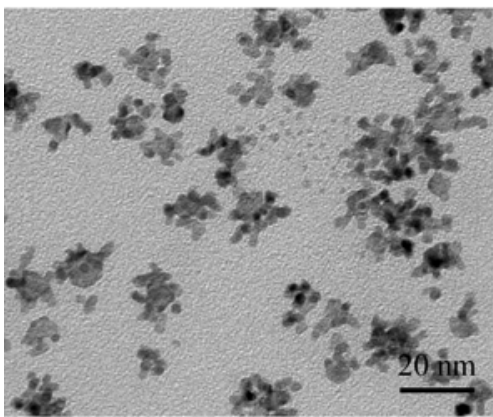

(c)

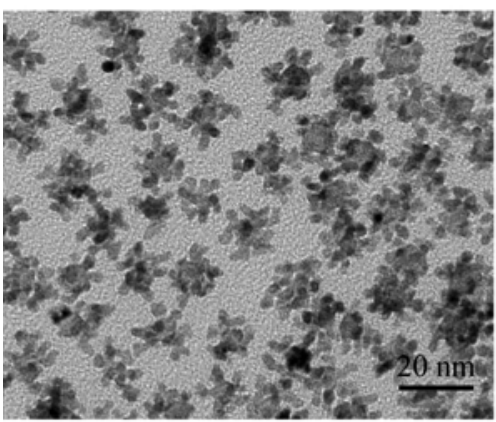

(d)

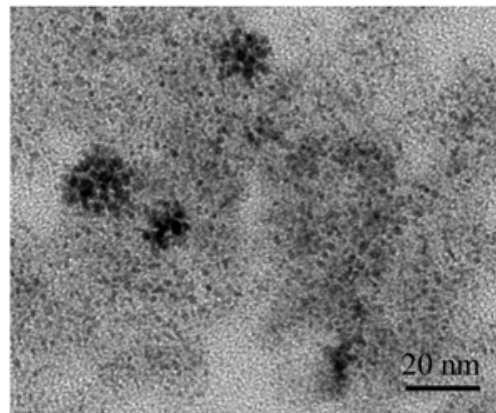

(e)

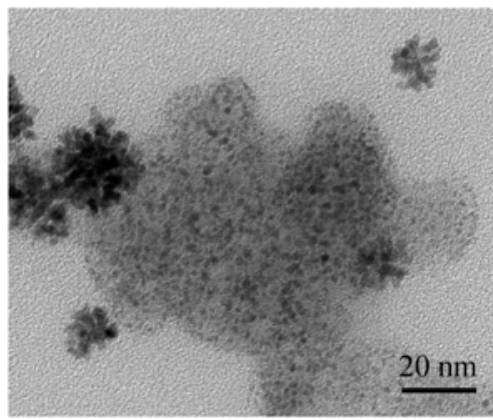

(f)

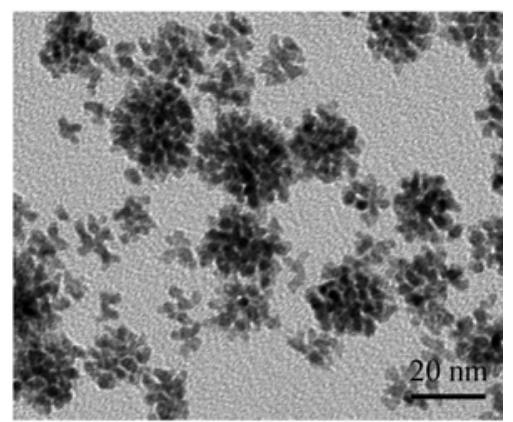

(g)

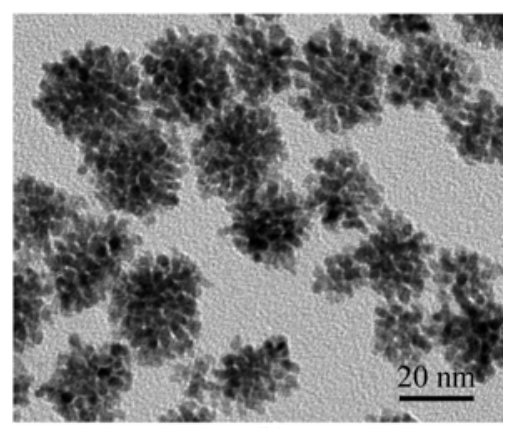

(h)
Figure 1 TEM images showing the morphological evolution of (a-d) Pd-Pt nanodendrites and $(\mathrm{e}-\mathrm{h})$ foam-like Pt aggregates synthesized in the presence and absence of Pd seeds, respectively. The reaction time was (a, e) $1 \mathrm{~min},(\mathrm{~b}, \mathrm{f}) 2 \mathrm{~min},(\mathrm{c}, \mathrm{g}) 5 \mathrm{~min}$, and $(\mathrm{d}, \mathrm{h}) 10 \mathrm{~min}$ 
Nano Res (2010) 3: 69-80

coherently extended from the $\mathrm{Pd}$ core to the $\mathrm{Pt}$ branches, suggesting that the Pt particles were epitaxially fused into the Pt bumps nucleated on the $\mathrm{Pd}$ seed. Most of the Pt branches exhibited a singlecrystal structure with a highly ordered, continuous fringe pattern, which supports the growth mechanism based on oriented attachment. However, we also found examples of the formation of a twin boundary at the attachment interface (Fig. 2(d)). In an oriented attachment process, twins can be formed when the particles on opposite sides of the interface are related by mirror symmetry [26]. High-resolution TEM analysis also revealed that the $\mathrm{Pt}$ branches were predominantly bounded by $\{111\}$ facets in addition to some $\{110\}$ facets (Fig. S-3 in the ESM).

Figures 3(a)-3(c) show high-resolution TEM images of a series of small $\mathrm{Pt}$ aggregates obtained at $5 \mathrm{~min}$ into the reaction carried out in the absence of $\mathrm{Pd}$ seeds. The aggregates had either a linear or a branched structure. The lattice fringes of these aggregates were perfectly aligned along the long axis, indicating that they were also formed via the oriented attachment mechanism. The similarity in morphology between the Pd-supported $\mathrm{Pt}$ branches and the small $\mathrm{Pt}$ aggregates strongly suggests that both nanostructures originated from the same growth mechanism based on oriented attachment. Although the initial $\mathrm{Pt}$ aggregates were defect free, the lattice fringes of the final, foam-like aggregates were not perfectly aligned across the entire particle (Fig. 3(d); also see Fig. S-4 in the ESM). This phenomenon is likely due to the involvement of twinning and/or imperfect oriented attachment characterized by a minor mis-orientation at the interface [28]. In the high-resolution TEM

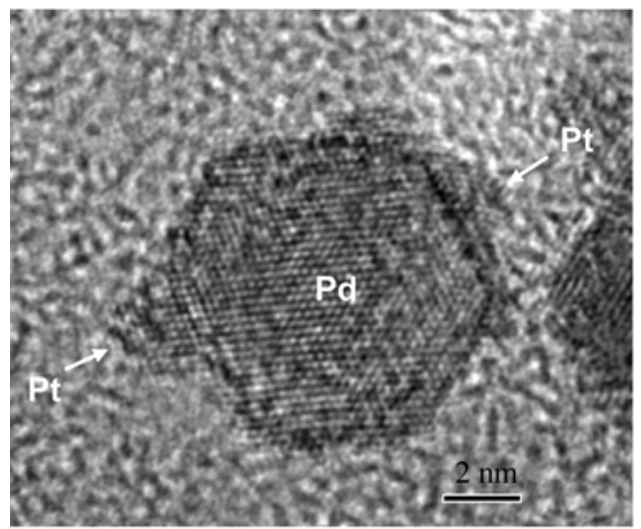

(a)

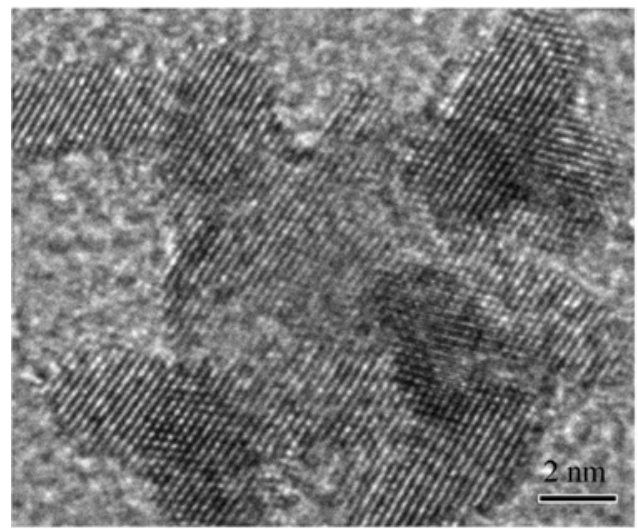

(c)

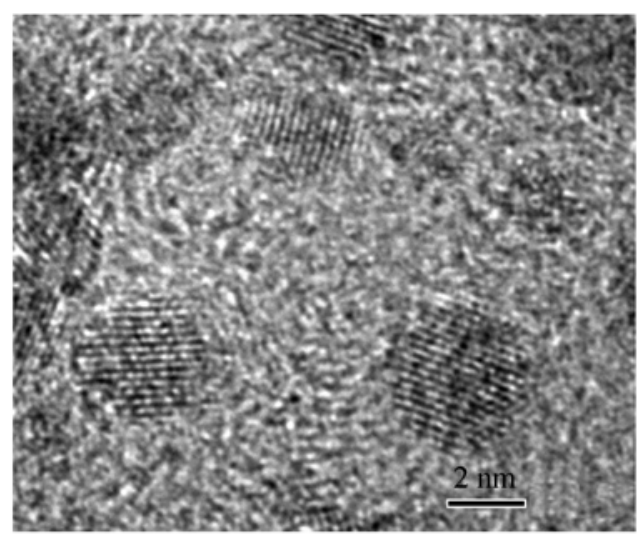

(b)

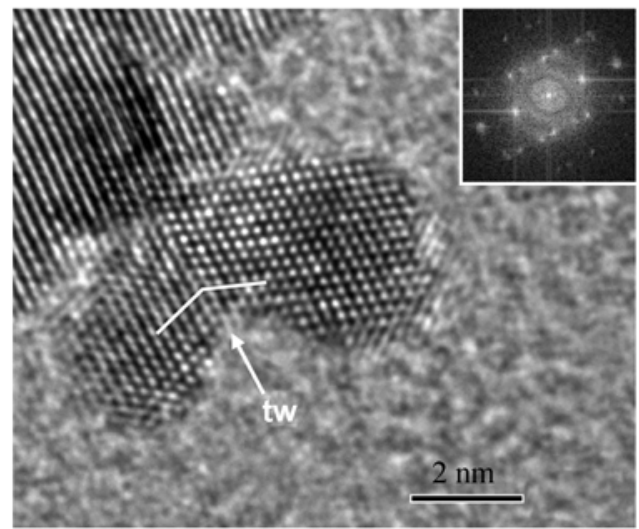

(d)

Figure 2 High-resolution TEM images of (a) an individual Pd-Pt particle and (b) the small Pt particles shown in Fig. 1(a). In (a), the Pt bumps on the Pd seed are indicated by arrows. (c) High-resolution TEM image of a single Pd-Pt nanodendrite. It can be seen that the lattice fringes are coherently extended from the Pd core to the Pt branches. (d) High-resolution TEM image taken from a Pt branch containing a single twin plane in its structure and the corresponding Fourier transform (FT) pattern (inset). A twin plane is indicated by tw 


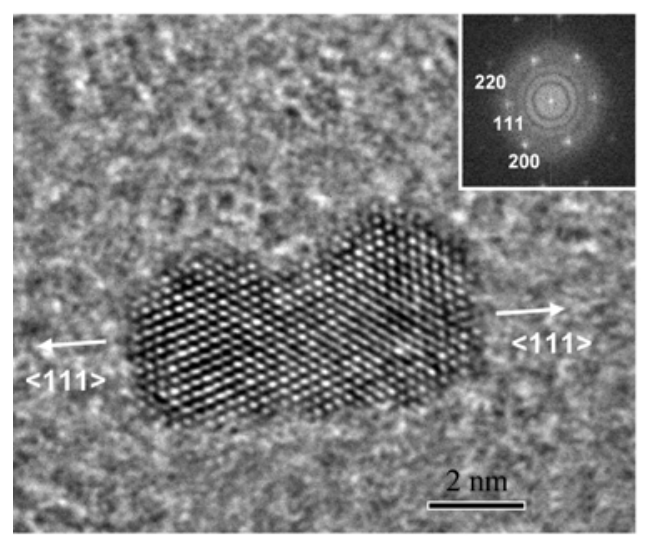

(a)

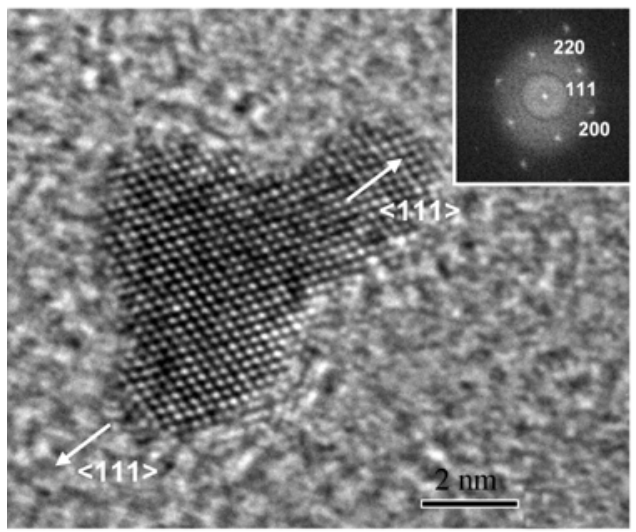

(c)

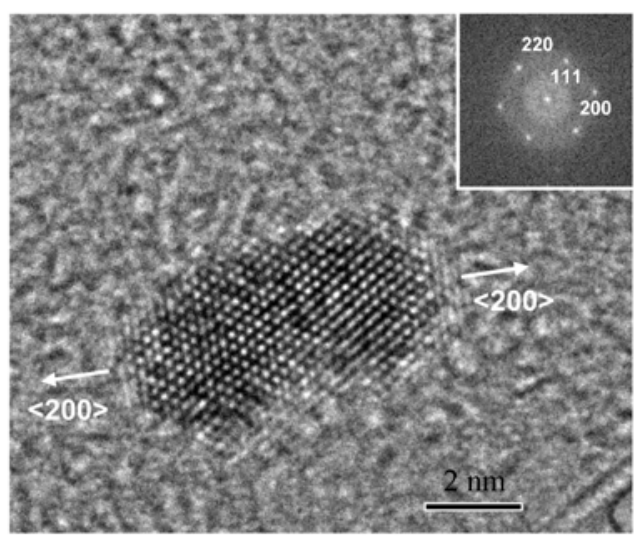

(b)

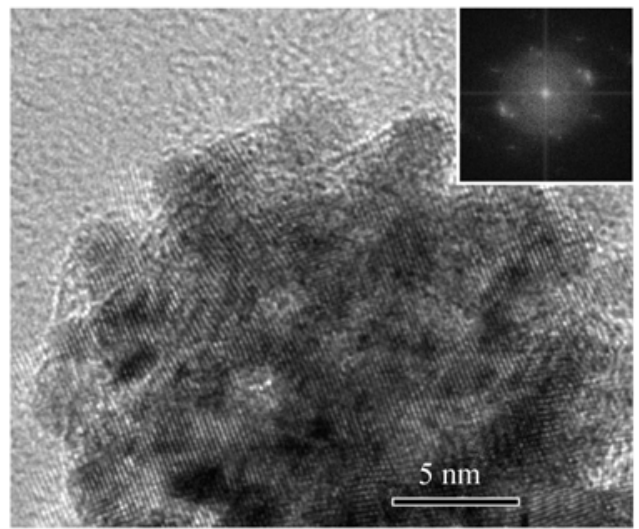

(d)

Figure 3 (a-c) High-resolution TEM images of small Pt aggregates obtained at 5 min into the reaction carried out in the absence of Pd seeds. (d) High-resolution TEM image of a foam-like Pt aggregate. The insets show the corresponding FT patterns

image, the foam-like Pt aggregates did not appear to have preferred facets on their surfaces.

In the absence of strong interparticle interactions (e.g., dipole-dipole interactions) [29-31] or a templating effect provided by self-assembled molecules $[32,33]$ the particles tend to aggregate in a diffusioncontrolled manner and accordingly dendritic growth can occur $[34,35]$. In previous studies, a mechanism based on slow nucleation and fast growth mediated by autocatalytic reduction has been proposed to explain the formation of foam-like Pt nanostructures prepared with $L$-ascorbic acid as a reducing agent in an aqueous solution [36,37]. As demonstrated in Figs. 1(e)-1(h), however, this type of nanostructures is a result of extensive self-aggregation, which has also been observed in the synthesis of nanoparticles of other metals such as $\mathrm{Ni}, \mathrm{Ru}$, and $\mathrm{Rh}$ [38-40]. The truncated octahedral $\mathrm{Pd}$ seeds play a pivotal role in achieving an open, dendritic structure by providing multiple nucleation sites for Pt. In this case, attachment is initiated at the $\mathrm{Pt}$ bumps on the $\mathrm{Pd}$ seed that are spatially separated from each other and, therefore, the Pt branches can grow without significant overlap and fusion between them. In this way, a large number of attachment sites provided by the growing Pd-Pt particles could also enable to avoid the formation of pure, foam-like Pt aggregates.

If two metals have a very close lattice match, the mode of heterogeneous nucleation is mainly determined by the interrelationship of the specific surface energies of the substrate $\left(\gamma_{s}\right)$, the deposit $\left(\gamma_{d}\right)$, and the substrate-deposit interface $\left(\gamma_{i}\right)$. This condition applies to the Pd-Pt system because Pd and Pt have a lattice mismatch of only $0.77 \%$. According to the thermodynamic analysis established by Bauer [41], the deposition proceeds in an island growth mode (Volmer-Weber mechanism) when $\gamma_{s}<\gamma_{d}+\gamma_{i}$, while the layered growth (Frank-van der Merwe mechanism) takes place when

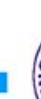


$\gamma_{s}=\gamma_{d}+\gamma_{i}$. Bauer's conditions predict an island growth mode for Pt deposition on a Pd surface because $\mathrm{Pt}$ has a relatively higher surface energy than Pd (Table S-1 in the ESM) [42], which is also consistent with our observation; that is, the formation of Pt bumps at multiple sites on the Pd seed. In a previous study, we observed the conformal growth of thin Pt layers on the surface of $\mathrm{Pd}$ nanoplates in the presence of citric acid as both the reducing and capping agent [5]. Citric acid seems to play a significant role in stabilizing the $\{111\}$ facets of the deposited Pt layers and lowering their surface energy, thus promoting the layered growth. In the current system, the stabilizing effect of PVP is relatively weak and, therefore, the layered growth is not favored.

Platinum is one of the most widely used elements in electrocatalysis because of its excellent properties in the adsorption and dissociation of hydrogen, oxygen, and various other molecules [43-48]. The performance of a Pt or Pt-based electrocatalyst is highly dependent upon the electrochemically active surface area (ECSA), which determines the number of catalytically active sites available for an electrochemical reaction. We first compared the ECSAs of the Pd-Pt nanodendrites and the foam-like Pt aggregates using cyclic voltammetry (CV). Figure 4(a) shows CV curves recorded at room temperature in $\mathrm{N}_{2}$-purged $0.1 \mathrm{~mol} / \mathrm{L} \mathrm{HClO}_{4}$ solutions with a sweep rate of $50 \mathrm{mV} / \mathrm{s}$. The ECSA was calculated by measuring the charge collected in the $\mathrm{H}_{\text {(upd) }}$ adsorption/desorption region after double-layer correction [49], where $\mathrm{H}_{\text {(upd) }}$ refers to the underpotentially deposited hydrogen. The ECSA was then normalized to the loading amount of metal in order to obtain the specific ECSA (i.e., ECSA per unit mass of metal). The specific ECSA of the Pd-Pt nanodendrites was $52.7 \mathrm{~m}^{2} / \mathrm{g}_{\mathrm{Pd}+\mathrm{Pt}}$, which was almost twice that of the foam-like $\mathrm{Pt}$ aggregates $\left(28.8 \mathrm{~m}^{2} / \mathrm{g}_{\mathrm{Pt}}\right)$. If the $\mathrm{Pt}$ mass was solely taken into account (85 wt.\% based on inductively coupled plasma mass spectrometry (ICP-MS) measurements), the specific ECSA of the $\mathrm{Pd}-\mathrm{Pt}$ nanodendrites $\left(62.0 \mathrm{~m}^{2} / \mathrm{g}_{\mathrm{Pt}}\right)$ was more than twice that of the foam-like Pt aggregates, demonstrating that the open dendritic structure provides a higher surface area.

We employed these two nanostructures as electrocatalysts for the ORR, the rate-determining step in a
PEM fuel cell [50]. The ORR measurements were performed at room temperature in $\mathrm{O}_{2}$-saturated $0.1 \mathrm{~mol} / \mathrm{L} \mathrm{HClO}_{4}$ solutions using a glassy carbon rotating disk electrode (RDE) with a sweep rate of $10 \mathrm{mV} / \mathrm{s}$ and a rotation rate of $1600 \mathrm{r} / \mathrm{min}$. Figure 4(b) presents ORR polarization curves for these two catalysts. The half-wave potentials for the Pd-Pt nanodendrites and the foam-like Pt aggregates were 867 and $834 \mathrm{mV}$ versus a reversible hydrogen electrode (RHE), respectively, indicating a higher ORR activity for the Pd-Pt nanodendrites. The kinetic current was calculated from the ORR polarization curve by using mass-transport correction and then normalized to the loading amount of metal in order to obtain the mass activity. Figure 4(c) shows a quantitative comparison of the mass activities of the $\mathrm{Pd}-\mathrm{Pt}$ nanodendrites and the foam-like Pt aggregates. In a mixed kineticdiffusion control region between 0.80 and $0.95 \mathrm{~V}$ versus $\mathrm{RHE}$, the $\mathrm{Pd}-\mathrm{Pt}$ nanodendrites outperformed the foam-like Pt aggregates by a factor of three on the basis of equivalent $\mathrm{Pt}$ mass.

In order to achieve a better understanding of the observed difference in ORR activity, we also compared the specific activities (i.e., kinetic current per unit surface area of catalyst) of these two catalysts. As illustrated in Fig. 4(d), the specific activity of the Pd-Pt nanodendrites was 1.4-1.6 times greater than that of the foam-like $\mathrm{Pt}$ aggregates in the potential region between 0.80 and $0.95 \mathrm{~V}$ versus RHE, indicating the accelerated ORR kinetics on the surface of the Pd-Pt nanodendrites. It is known that the difference in ORR activity on low-index Pt surfaces most likely arises from the structure-sensitive inhibiting effect of $\mathrm{OH}_{(\mathrm{ad})}$ species on $\mathrm{Pt}(h k l)$ and, in a non-adsorbing electrolyte such as perchloric acid, the activity increases on the order of $\operatorname{Pt}(100)<<\operatorname{Pt}(111)<\operatorname{Pt}(110)$, with the difference in activity between $\mathrm{Pt}(111)$ and $\mathrm{Pt}(110)$ being quite small [51]. For the $\mathrm{Pd}-\mathrm{Pt}$ nanodendrites, the preferential exposure of $\{111\}$ facets in addition to some $\{110\}$ facets on the Pd-supported Pt branches may account for their higher specific activity as compared to the foam-like Pt aggregates with poorly-defined surface facets.

The Pd-Pt nanodendrites have also been evaluated as electrocatalysts for the oxidation of formic acid. We performed potentiodynamic experiments in order 


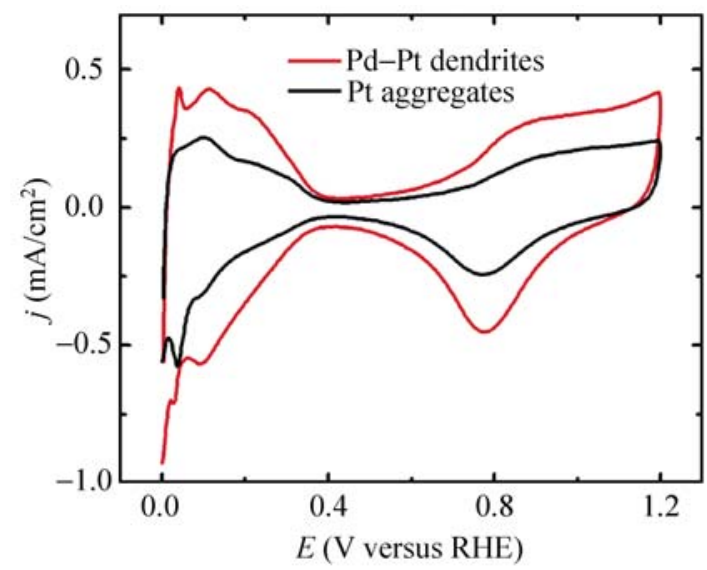

(a)

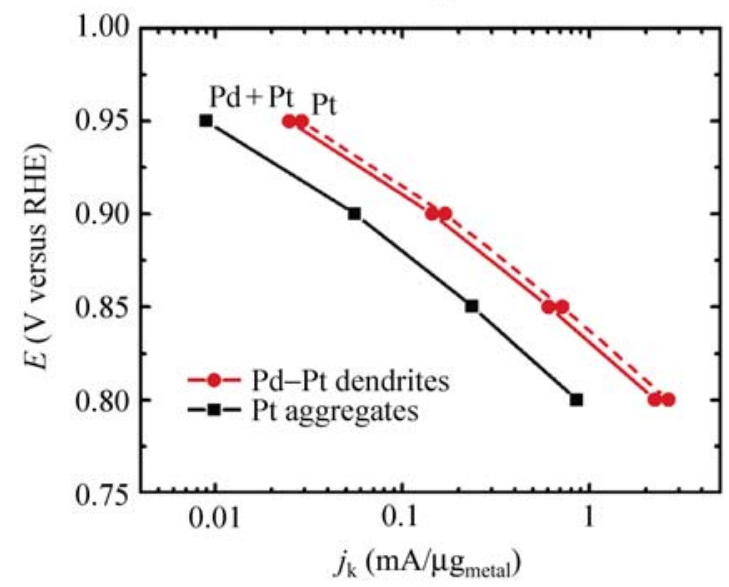

(c)

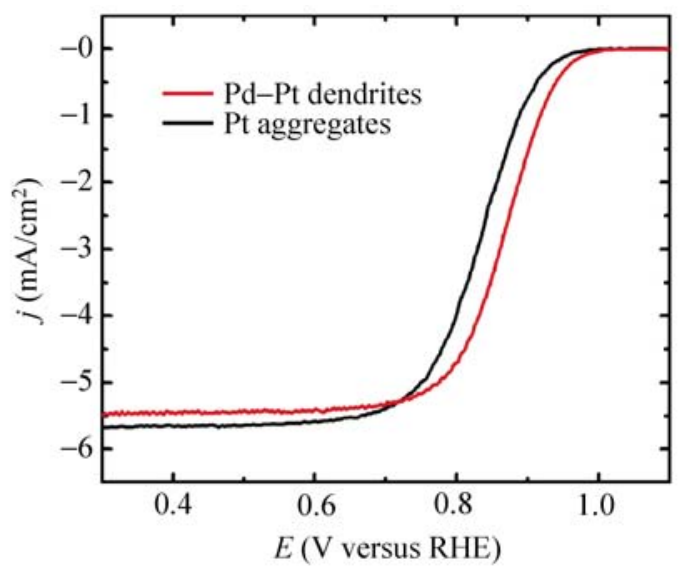

(b)

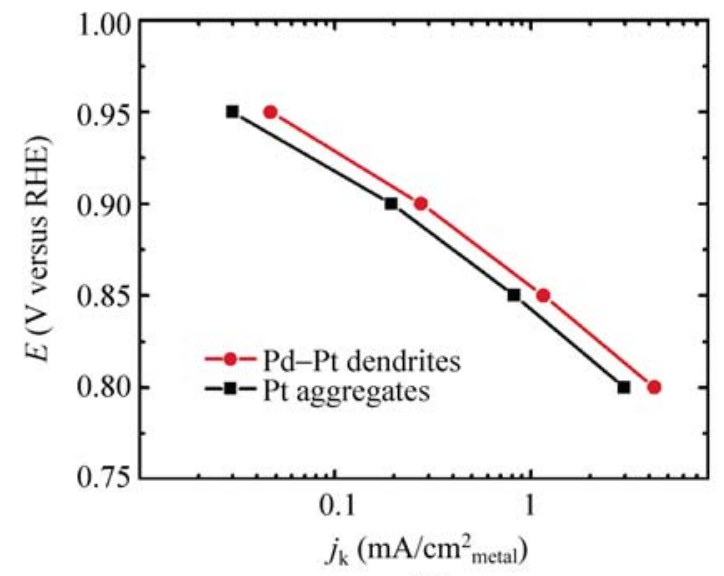

(d)

Figure 4 Comparison of electrocatalytic properties of the Pd-Pt nanodendrites and foam-like Pt aggregates for the ORR: (a) CV curves recorded at room temperature in $\mathrm{N}_{2}$-purged $0.1 \mathrm{~mol} / \mathrm{L} \mathrm{HClO}_{4}$ solutions at a sweep rate of $50 \mathrm{mV} / \mathrm{s}$; (b) ORR polarization curves recorded at room temperature in $\mathrm{O}_{2}$-saturated $0.1 \mathrm{~mol} / \mathrm{L} \mathrm{HClO}_{4}$ solutions at a sweep rate of $10 \mathrm{mV} / \mathrm{s}$ and a rotation rate of $1600 \mathrm{r} / \mathrm{min}$; (c, d) Mass and specific activities given as kinetic current densities $\left(j_{\mathrm{k}}\right)$ normalized against the loading amount of metal and the ECSA of catalyst, respectively (red solid line is the activity based on the total mass of Pd and Pt (i.e., Pd $+\mathrm{Pt}$ ); red break line is the activity based on the mass of Pt). For all electrodes, the metal loading on a glassy carbon electrode was $15.3 \mu \mathrm{g} / \mathrm{cm}^{2}$. In (a) and (b), the current densities were normalized against the geometric area of the electrode $\left(0.196 \mathrm{~cm}^{2}\right)$

to investigate the individual reaction steps and adsorbed intermediates involved in the formic acid oxidation. Figure 5(a) shows CV curves for the formic acid oxidation catalyzed by the Pd-Pt nanodendrites and the foam-like $\mathrm{Pt}$ aggregates. For the foam-like $\mathrm{Pt}$ aggregates, only a minimal current associated with formic acid oxidation was observed between 0.30 and $0.70 \mathrm{~V}$ in the positive scan, implying that the surface was largely deactivated by the formation of a self-poisoning species (e.g., $\mathrm{CO}_{(\mathrm{ad})}$ ). The oxidative elimination of the adsorbed $\mathrm{CO}$ at higher overpotentials $(>0.80 \mathrm{~V})$ regenerates a bare $\mathrm{Pt}$ surface, which accounts for the higher currents observed in the negative scan. On the other hand, the $\mathrm{Pd}-\mathrm{Pt}$ nanodendrites showed less poisoning in the positive scan, with relatively higher currents between 0.30 and $0.70 \mathrm{~V}$, suggesting that they provide a direct path for formic acid oxidation that does not form a selfpoisoning species.

For a quantitative assessment of electrocatalytic activity, potentiostatic experiments were conducted, in which the potential was stepped to a target potential that was varied from 0.55 to $0.70 \mathrm{~V}$ and held for $60 \mathrm{~s}$ to obtain a steady-state current. Figure 5(b) shows transient current density curves for the formic acid oxidation on the $\mathrm{Pd}-\mathrm{Pt}$ nanodendrites and the 


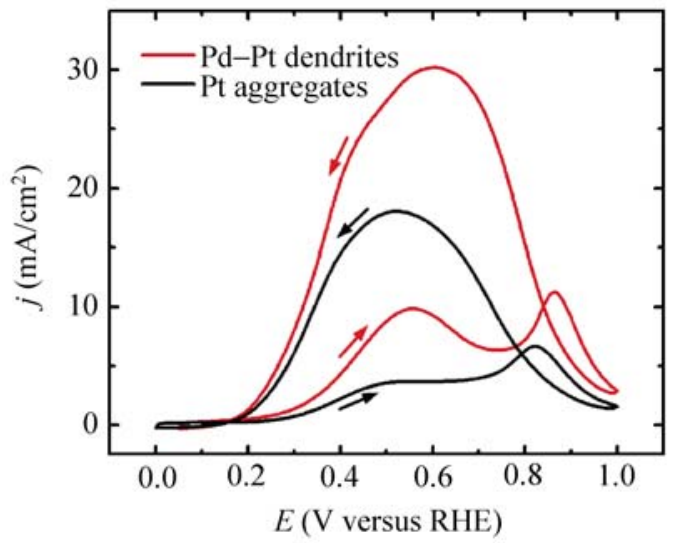

(a)

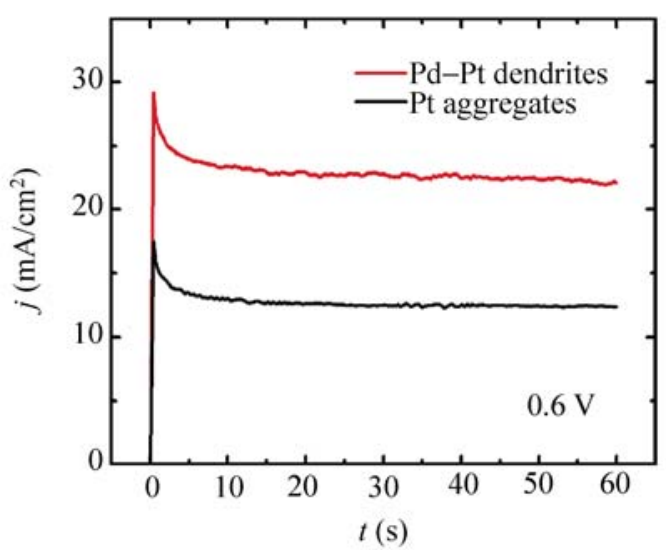

(b)

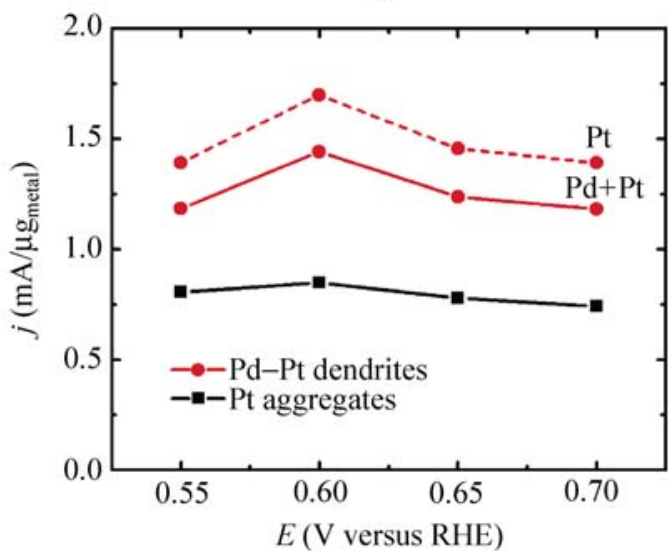

(c)

Figure 5 Comparison of electrocatalytic properties of the $\mathrm{Pd}-\mathrm{Pt}$ nanodendrites and foam-like Pt aggregates for the formic acid oxidation reaction: (a) $\mathrm{CV}$ curves recorded at room temperature in $0.25 \mathrm{~mol} / \mathrm{L} \mathrm{HCOOH}+0.5 \mathrm{~mol} / \mathrm{L} \mathrm{H}_{2} \mathrm{SO}_{4}$ solutions with a sweep rate of $50 \mathrm{mV} / \mathrm{s}$; (b) transient current density curves at $0.6 \mathrm{~V}$ versus RHE; (c) mass activities given as the steady-state current densities (recorded at $60 \mathrm{~s}$ ) normalized against the loading amount of metal (red solid line is the activity based on the total mass of $\mathrm{Pd}$ and $\mathrm{Pt}$ (i.e., $\mathrm{Pd}+\mathrm{Pt}$ ); red break line is the activity based on the mass of $\mathrm{Pt}$ ). For all electrodes, the metal loading on a glassy carbon electrode was $15.3 \mu \mathrm{g} / \mathrm{cm}^{2}$. In (a) and (b), the current densities were normalized against the geometric area of the electrode $\left(0.196 \mathrm{~cm}^{2}\right)$ foam-like Pt aggregates recorded at $0.60 \mathrm{~V}$. The steadystate current recorded at $60 \mathrm{~s}$ has been normalized to the loading amount of metal in order to compare the mass activity. As shown in Fig. 5(c), the Pd-Pt nanodendrites exhibited higher mass activities than the foam-like $\mathrm{Pt}$ aggregates in the potential region of $0.55-0.70 \mathrm{~V}$ versus RHE. At a peak potential of $0.60 \mathrm{~V}$ versus $\mathrm{RHE}$, the $\mathrm{Pd}-\mathrm{Pt}$ nanodendrites exhibited a two-fold improvement in Pt mass activity over the foam-like Pt aggregates.

In the electro-oxidation of formic acid, $\mathrm{CO}_{(\mathrm{ad})}$ formed by dehydration of formic acid has been identified as the main poisoning species on Pt surfaces. Formic acid oxidation on Pt surfaces proceeds via the so-called dual-path mechanism as shown in the following reaction schemes [52]:

$$
\begin{gathered}
\mathrm{HCOOH}_{(\mathrm{ad})} \rightarrow \mathrm{HCOO}_{(\mathrm{ad})}+\mathrm{H}^{+}+\mathrm{e}^{-} \rightarrow \mathrm{CO}_{2}+2 \mathrm{H}^{+}+2 \mathrm{e}^{-} \\
\mathrm{HCOOH}_{(\mathrm{ad})} \rightarrow \mathrm{CO}_{(\mathrm{ad})}+\mathrm{H}_{2} \mathrm{O} \\
\mathrm{CO}_{(\mathrm{ad})}+\mathrm{OH}_{(\mathrm{ad})} \rightarrow \mathrm{CO}_{2}+\mathrm{H}^{+}+\mathrm{e}^{-}
\end{gathered}
$$

The direct path involves the dehydrogenation of formic acid to $\mathrm{CO}_{2}$ (reaction 1), while a dehydration reaction produces $\mathrm{CO}_{(\text {ad) }}$ and $\mathrm{H}_{2} \mathrm{O}$ (reaction 2). The adsorbed CO may have two modes of action on the formic acid oxidation depending on the overpotential. At relatively low overpotentials, $\mathrm{CO}_{(\mathrm{ad})}$ acts as a poison and thus impedes the formic acid oxidation, whereas at higher overpotentials (typically $>0.80 \mathrm{~V}$ versus RHE) $\mathrm{CO}_{(\mathrm{ad})}$ can serve as a reactive intermediate by reacting with $\mathrm{OH}_{(\mathrm{ad})}$ formed from the dissociation of water and thus provide a reaction channel to $\mathrm{CO}_{2}$ (reaction 3). It has been shown that for the formic acid oxidation on low-index $\mathrm{Pt}$ surfaces, the dehydrogenation and dehydration pathways are predominant on the $\operatorname{Pt}(111)$ and $\operatorname{Pt}(100)$ surfaces, respectively, while the $\operatorname{Pt}(110)$ surface exhibits an intermediate behavior [53]. The predominant exposure of $\{111\}$ facets on the Pt branches of the Pd-Pt nanodendrites could allow the direct dehydrogenation path for formic acid oxidation and thus lead to reduced poisoning by $\mathrm{CO}_{(\text {ad) }}$ species, which may contribute to their higher activity. 
In summary, we have resolved the nucleation and growth mechanisms involved in the seed-mediated synthesis of $\mathrm{Pd}-\mathrm{Pt}$ bimetallic nanodendrites. Our results indicate that both homogeneous and heterogeneous nucleation of Pt occurred at very early stages of the synthesis and the growth proceeded via particle attachment. The Pd seeds played a crucial role in forming an open, dendritic structure by providing multiple nucleation sites for $\mathrm{Pt}$ that were spatially separated from each other, helping to avoid overlap and fusion between the Pt branches during the growth process. In the absence of Pd seeds, extensive self-aggregation of small $\mathrm{Pt}$ particles led to the formation of Pt nanostructures with a foam-like morphology. Due to their open, dendritic structure, the Pd-Pt nanodendrites exhibited a higher surface area and much enhanced activity for both the oxygen reduction and formic acid oxidation reactions as compared to the foam-like Pt aggregates. This work not only greatly advances our understanding of the dendritic growth of $\mathrm{Pt}$ nanostructures but also provides new insights into the mechanisms involved in heterogeneous, seeded growth. It is worth pointing out that foam-like $\mathrm{Pt}$ nanostructures have been reported by a number of groups (including us) and suggested as a promising candidate for a fuel cell electrocatalyst. The comparative studies described herein clearly contradict that suggestion. Our results show that a synthetic methodology based on heterogeneous, seeded growth provides a simple and more efficient way for generating Pt-based electrocatalysts with improved activities.

\section{Experimental}

Synthesis of Pd-Pt nanodendrites. For the preparation of 9-nm truncated octahedral Pd seeds, $11 \mathrm{~mL}$ of an aqueous solution containing PVP $(M w \approx 55000$, $105 \mathrm{mg}$, Aldrich), L-ascorbic acid (60 mg, Aldrich), citric acid (60 mg, Fisher), and $\mathrm{Na}_{2} \mathrm{PdCl}_{4}(57 \mathrm{mg}$, Aldrich) was heated at $100{ }^{\circ} \mathrm{C}$ in air under magnetic stirring for $3 \mathrm{~h}$ and cooled down to room temperature [54]. The Pd-Pt nanodendrites were prepared using a previously reported method [12]. In a typical synthesis, $1 \mathrm{~mL}$ of the as-prepared suspension of the $\mathrm{Pd}$ seeds and $6 \mathrm{~mL}$ of an aqueous solution containing PVP
(35 mg) and $L$-ascorbic acid $(60 \mathrm{mg})$ were added into a 25-mL, three-necked flask. The mixture was heated to $90{ }^{\circ} \mathrm{C}$ in air under magnetic stirring. Meanwhile, $\mathrm{K}_{2} \mathrm{PtCl}_{4}$ (27 mg, Aldrich) was dissolved in $3 \mathrm{~mL}$ of deionized water at room temperature. The aqueous $\mathrm{K}_{2} \mathrm{PtCl}_{4}$ solution was then rapidly injected into the flask using a pipette. The reaction mixture was heated at $90{ }^{\circ} \mathrm{C}$ in air for $3 \mathrm{~h}$, and then cooled down to room temperature. The product was collected by centrifugation and washed several times with ethanol and deionized water for further use in electrochemical measurements.

Samples for TEM and high-resolution TEM. In the preparation of samples for TEM and high-resolution TEM studies, aliquots of the reaction solution were rapidly cooled by adding pre-cooled acetone. The resultant solutions were dropped directly onto carboncoated copper grids without centrifugation and dried inside the vacuum chamber of a freeze-drier. After drying, the grids were washed with ethanol to remove excess PVP. TEM images were captured using a Phillips 420 microscope operated at $120 \mathrm{kV}$. High-resolution TEM was carried out on a JEOL 2100F microscope operated at $200 \mathrm{kV}$.

Electrochemical measurements. Electrochemical measurements were performed using a glassy carbon RDE (Pine Research Instrumentation) connected to a PARSTAT 283 potentiostat (Princeton Applied Research). A leak-free $\mathrm{AgCl} / \mathrm{Ag} / \mathrm{KCl}$ (3 mol/L) electrode (Warner Instrument) was used as the reference. The counter electrode was a platinum mesh $(1 \mathrm{~cm} \times 1 \mathrm{~cm})$ attached to a platinum wire. All potentials were converted to values with reference to RHE. To prepare the working electrode, the sample was diluted to $0.15 \mu \mathrm{g} / \mu \mathrm{L}$ (based on ICP-MS measurements) and $20 \mu \mathrm{L}$ of the dispersion was transferred onto the glassy carbon RDE with a geometric area of $0.196 \mathrm{~cm}^{2}$. Therefore, the loading amount of metal for Pd-Pt nanodendrites and foam-like Pt aggregates was $3 \mu \mathrm{g}$ (i.e., $15.3 \mu \mathrm{g} / \mathrm{cm}^{2}$ based on the geometric electrode area). After water evaporation, the electrode was covered with $15 \mu \mathrm{L}$ of 0.05 wt.\% Nafion solution. In order to produce a clean electrode surface, several potential sweeps between 0.0 and $1.2 \mathrm{~V}$ versus RHE were applied to the electrode prior to the measurement. 
$\mathrm{CV}$ measurements were carried out in $0.1 \mathrm{~mol} / \mathrm{L}$ $\mathrm{HClO}_{4}$ solutions under a flow of $\mathrm{N}_{2}$ (Airgas, ultrahigh purity) at a sweep rate of $50 \mathrm{mV} / \mathrm{s}$. The ECSA was estimated by measuring the charge associated with $\mathrm{H}_{\text {(upd) }}$ adsorption $\left(Q_{\mathrm{H}}\right)$ between 0.05 and $0.40 \mathrm{~V}$ and assuming a value of $210 \mu \mathrm{C} / \mathrm{cm}^{2}$ for the adsorption of a monolayer of hydrogen on a Pt surface $\left(q_{\mathrm{H}}\right)$. The $\mathrm{H}_{\text {(upd) }}$ adsorption charge $\left(Q_{\mathrm{H}}\right)$ can be determined using $Q_{\mathrm{H}}=0.5 \times Q$, where $Q$ is the charge in the $\mathrm{H}_{\text {(upd) }}$ adsorption/desorption area obtained after double-layer correction. Then, the specific ECSA was calculated based on the following relation: specific ECSA $=Q_{\mathrm{H}} /\left(m \times q_{\mathrm{H}}\right)$, where $Q_{\mathrm{H}}$ is the charge for $\mathrm{H}_{\text {(upd) }}$ adsorption, $m$ is the loading amount of metal, and $q_{\mathrm{H}}$ is the charge required for the adsorption of a monolayer of hydrogen on a Pt surface.

The ORR measurements were performed at room temperature in $0.1 \mathrm{~mol} / \mathrm{L} \mathrm{HClO}_{4}$ solutions under a flow of $\mathrm{O}_{2}$ (Airgas, Research grade) using the glassy carbon $\mathrm{RDE}$ at a rotation rate of $1600 \mathrm{r} / \mathrm{min}$ and a sweep rate of $10 \mathrm{mV} / \mathrm{s}$. The kinetic current was calculated based on the Koutecky-Levich equation as follows:

$$
\frac{1}{i}=\frac{1}{i_{\mathrm{k}}}+\frac{1}{i_{\mathrm{d}}}
$$

where $i$ is the experimentally measured current, $i_{\mathrm{d}}$ is the diffusion-limiting current, and $i_{\mathrm{k}}$ is the kinetic current. For each catalyst, the kinetic current was normalized to the loading amount of the metal and the ECSA in order to obtain mass and specific activities, respectively.

The electrochemical measurements for the formic acid oxidation reaction were performed at room temperature in a solution containing $0.25 \mathrm{~mol} / \mathrm{L}$ $\mathrm{HCOOH}$ and $0.5 \mathrm{~mol} / \mathrm{L} \mathrm{H}_{2} \mathrm{SO}_{4}$. CV curves were collected at a sweep rate of $50 \mathrm{mV} / \mathrm{s}$. In the potentiostatic experiments, the potential was stepped from $0.0 \mathrm{~V}$ to the high potential that varies from 0.55 to $0.70 \mathrm{~V}$, and the transient current density curve of the formic acid oxidation was recorded.

\section{Acknowledgements}

This work was supported in part by the National Science Foundation (NSF) (No. DMR-0804088) and startup funds from Washington University in St. Louis. T.Y. was also partially supported by the National Research Foundation of Korea Grant funded by the Korean Government (No. NRF-2009-352-D00160). Pedro Henrique Cury Camargo (P.H.C.C.) was also partially supported by the Fulbright Program and the Brazilian Ministry of Education (CAPES). Part of the work was performed at the Nano Research Facility (NRF), a member of the National Nanotechnology Infrastructure Network (NNIN), which is supported by the National Science Foundation under award No. ECS-0335765. NRF is part of the School of Engineering and Applied Science at Washington University in St. Louis.

Electronic Supplementary Material: Additional TEM and high-resolution TEM images (Figs. S-1 to S-4) and the surface energies of $\mathrm{Pd}$ and $\mathrm{Pt}$ (Table S-1) are available in the online version of this article at http://dx.doi.org/10.1007/s12274-010-1010-8 and are accessible free of charge.

\section{References}

[1] Tsuji, M.; Miyamae, N.; Lim, S.; Kimura, K.; Zhang, X.; Hikino, S.; Nishio, M. Crystal structures and growth mechanisms of $\mathrm{Au} @ \mathrm{Ag}$ core-shell nanoparticles prepared by the microwave-polyol method. Cryst. Growth Des. 2006, 6, 1801-1807.

[2] Habas, S. E.; Lee, H.; Radmilovic, V.; Somorjai, G. A.; Yang, P. Shaping binary metal nanocrystals through epitaxial seeded growth. Nat. Mater. 2007, 6, 692-697.

[3] Fan, F. R.; Liu, D. Y.; Wu, Y. F.; Duan, S.; Xie, Z. X.; Jiang, Z. Y.; Tian, Z. Q. Epitaxial growth of heterogeneous metal nanocrystals: From gold nano-octahedra to palladium and silver nanocubes. J. Am. Chem. Soc. 2008, 130, 6949-6951.

[4] Xue, C.; Millstone, J. E.; Li, S.; Mirkin, C. A. Plasmondriven synthesis of triangular core-shell nanoprisms from gold seeds. Angew. Chem. Int. Ed. 2007, 46, 8436-8439.

[5] Lim, B.; Wang, J.; Camargo, P. H. C.; Jiang, M.; Kim, M. J.; $\mathrm{Xia}, \mathrm{Y}$. Facile synthesis of bimetallic nanoplates consisting of Pd cores and Pt shells through seeded epitaxial growth. Nano Lett. 2008, 8, 2535-2540.

[6] Tsuji, M.; Matsuo, R.; Jiang, P.; Miyamae, N.; Ueyama, D.; Nishio, M.; Hikino, S.; Kumagae, H.; Kamarudin, K. S. N.; Tang, X. L. Shape-dependent evolution of Au@Ag coreshell nanocrystals by PVP-assisted $N, N$-dimethylformamide reduction. Cryst. Growth Des. 2008, 8, 2528-2536. 
[7] Camargo, P. H. C.; Xiong, Y.; Ji, L.; Zuo, J. M.; Xia, Y. Facile synthesis of tadpole-like nanostructures consisting of $\mathrm{Au}$ heads and Pd tails. J. Am. Chem. Soc. 2007, 129, 15452-15453.

[8] Seo, D.; Yoo, C. I.; Jung, J.; Song, H. Ag-Au-Ag heterometallic nanorods formed through directed anisotropic growth. J. Am. Chem. Soc. 2008, 130, 2940-2941.

[9] Park, K.; Vaia, R. A. Synthesis of complex Au/Ag nanorods by controlled overgrowth. Adv. Mater. 2008, 20, 3882-3886.

[10] Zhou, S.; McIlwrath, K.; Jackson, G.; Eichhorn, B. Enhanced $\mathrm{CO}$ tolerance for hydrogen activation in $\mathrm{Au}-\mathrm{Pt}$ dendritic heteroaggregate nanostructures. J. Am. Chem. Soc. 2006, 128, 1780-1781.

[11] Peng, Z.; Yang, H. PtAu bimetallic heteronanostructures made by post-synthesis modification of Pt-on-Au nanoparticles. Nano Res. 2009, 2, 406-415.

[12] Lim, B.; Jiang, M.; Camargo, P. H. C.; Cho, E. C.; Tao, J.; Lu, X.; Zhu, Y.; Xia, Y. Pd-Pt bimetallic nanodendrites with high activity for oxygen reduction. Science 2009, 324, 1302-1305.

[13] Peng, Z; Yang, H. Synthesis and oxygen reduction electrocatalytic property of Pt-on-Pd bimetallic heteronanostructures. J. Am. Chem. Soc. 2009, 131, 7542-7543.

[14] Cacciuto, A.; Auer, S.; Frenkel, D. Onset of heterogeneous crystal nucleation in colloidal suspensions. Nature 2004, 428, 404-406.

[15] Auer, S.; Frenkel, D. Prediction of absolute crystalnucleation rate in hard-sphere colloids. Nature 2001, 409, 1020-1023.

[16] Park, J.; Joo, J.; Kwon, S. G.; Jang, Y.; Hyeon, T. Synthesis of monodisperse spherical nanocrystals. Angew. Chem. Int. Ed. 2007, 46, 4630-4660.

[17] Pastoriza-Santos, I.; Liz-Marzán, L. M. Formation of PVP-protected metal nanoparticles in DMF. Langmuir 2002, 18, 2888-2894.

[18] Shin, H. S.; Yang, H. J.; Kim, S. B.; Lee, M. S. Mechanism of growth of colloidal silver nanoparticles stabilized by polyvinyl pyrrolidone in gamma-irradiated silver nitrate solution. J. Colloid Interface Sci. 2004, 274, 89-94.

[19] Ewers, T. D.; Sra, A. K.; Norris, B. C.; Cable, R. E.; Cheng, C. H.; Shantz, D. F.; Schaak, R. E. Spontaneous hierarchical assembly of rhodium nanoparticles into spherical aggregates and superlattices. Chem. Mater. 2005, 17, 514-520.

[20] Banfield, J. F.; Welch, S. A.; Zhang, H.; Ebert, T. T.; Penn, R. L. Aggregation-based crystal growth and microstructure development in natural iron oxyhydroxide biomineralization products. Science 2000, 289, 751-754.

[21] Pacholski, C.; Kornowski, A.; Weller, H. Self-assembly of $\mathrm{ZnO}$ : From nanodots to nanorods. Angew. Chem. Int. Ed.
2002, 41, 1188-1191.

[22] Huang, F.; Zhang, H.; Banfield, J. F. The role of oriented attachment crystal growth in hydrothermal coarsening of nanocrystalline ZnS. J. Phys. Chem. B 2003, 107, 10470-10475.

[23] Niederberger, M.; Cölfen, H. Oriented attachment and mesocrystals: Non-classical crystallization mechanisms based on nanoparticle assembly. Phys. Chem. Chem. Phys. 2006, 8, 3271-3287.

[24] Yu, J. H.; Joo, J.; Park, H. M.; Baik, S. I.; Kim, Y. W.; Kim, S. C.; Hyeon, T. Synthesis of quantum-sized cubic $\mathrm{ZnS}$ nanorods by the oriented attachment mechanism. J. Am. Chem. Soc. 2005, 127, 5662-5670.

[25] Ji, X.; Song, X.; Li, J.; Bai, Y.; Yang, W.; Peng, X. Size control of gold nanocrystals in citrate reduction: The third role of citrate. J. Am. Chem. Soc. 2007, 129, 13939-13948.

[26] Halder, A.; Ravishankar, N. Ultrafine single-crystalline gold nanowire arrays by oriented attachment. Adv. Mater. 2007, 19, 1854-1858.

[27] Bisson, L.; Boissiere, C.; Nicole, L.; Grosso, D.; Jolivet, J. P.; Thomazeau, C.; Uzio, D.; Berhault, G.; Sanchez, C. Formation of palladium nanostructures in a seed-mediated synthesis through an oriented-attachment-directed aggregation. Chem. Mater. 2009, 21, 2668-2678.

[28] Penn, R. L.; Banfield, J. F. Imperfect oriented attachment: Dislocation generation in defect-free nanocrystals. Science 1998, 281, 969-971.

[29] Tang, Z.; Kotov, N. A.; Giersig, M. Spontaneous organization of single CdTe nanoparticles into luminescent nanowires. Science 2002, 297, 237-240.

[30] Tang, Z.; Zhang, Z.; Wang, Y.; Glotzer, S. C.; Kotov, N. A. Self-assembly of CdTe nanocrystals into free-floating sheets. Science 2006, 314, 274-278.

[31] Zhang, Z.; Tang, Z.; Kotov, N. A.; Glotzer, S. C. Simulations and analysis of self-assembly of $\mathrm{CdTe}$ nanoparticles into wires and sheets. Nano Lett. 2007, 7, 1670-1675.

[32] Warren, S. C.; Messina, L. C.; Slaughter, L. S.; Kamperman, M.; Zhou, Q.; Gruner, S. M.; DiSalvo, F. J.; Wiesner, U. Ordered mesoporous materials from metal nanoparticleblock copolymer self-assembly. Science 2008, 320, 1748-1752.

[33] Sharma, J.; Chhabra, R.; Cheng, A.; Brownell, J.; Liu, Y.; Yan, H. Control of self-assembly of DNA tubules through integration of gold nanoparticles. Science 2009, 323, 112-116.

[34] Witten, T. A.; Sander, L. M. Diffusion-limited aggregation, a kinetic critical phenomenon. Phys. Rev. Lett. 1981, 47, 1400-1403. 
[35] Meakin, P.; Stanley, H. E. Spectral dimension for the diffusion-limited aggregation model of colloid growth. Phys Rev. Lett. 1983, 51, 1457-1460.

[36] Song, Y.; Yang, Y. Medforth, C. J.; Pereira, E.; Singh, A. K.; Xu, H.; Jiang, Y.; Brinker, C. J.; Swol, F.; Shelnutt, J. A. Controlled synthesis of 2-D and 3-D dendritic platinum nanostructures. J. Am. Chem. Soc. 2004, 126, 635-645.

[37] Wang, L.; Yamauchi, Y. Facile synthesis of threedimensional dendritic platinum nanoelectrocatalyst. Chem. Mater. 2009, 21, 3562-3569.

[38] Vidoni, O.; Philippot, K.; Amiens, C.; Chaudret, B.; Balmes, O.; Malm, J. O.; Bovin, J. O.; Senocq, F.; Casanove, M. J. Novel, spongelike ruthenium particles of controllable size stabilized only by organic solvents. Angew. Chem. Int. Ed. 1999, 38, 3736-3738.

[39] Ely, T. O.; Amiens, C.; Chaudret, B. Synthesis of nickel nanoparticles. Influence of aggregation induced by modification of poly(vinylpyrrolidone) chain length on their magnetic properties. Chem. Mater. 1999, 11, 526-529.

[40] Pelzer, K.; Vidoni, O.; Philippot, K.; Chaudret, B.; Collière, V. Organometallic synthesis of size-controlled polycrystalline ruthenium nanoparticles in the presence of alcohols. $A d v$. Funct. Mater. 2003, 13, 118-126.

[41] Bauer, E.; Poppa, H. Recent advances in epitaxy. Thin Solid Films 1972, 12, 167-185.

[42] Jiang, Q.; Lu, H. M.; Zhao, M. Modelling of surface energies of elemental crystals. J. Phys.: Condens. Matter 2004, 16, 521-530.

[43] Kua, J.; Goddard, W. A. Oxidation of methanol on 2nd and 3rd row group VIII transition metals ( $\mathrm{Pt}, \mathrm{Ir}, \mathrm{Os}, \mathrm{Pd}, \mathrm{Rh}$, and $\mathrm{Ru}$ ): Application to direct methanol fuel cells. J. Am. Chem. Soc. 1999, 121, 10928-10941.

[44] Nørskov, J. K.; Rossmeisl, J.; Logadottir, A.; Lindqvist, L.; Kitchin, J. R.; Bligaard, T.; Jónsson, H. Origin of the overpotential for oxygen reduction at a fuel-cell cathode. $J$. Phys. Chem. B 2004, 108, 17886-17892.
[45] Teng, X.; Liang, X.; Maksimuk, S.; Yang, H. Synthesis of porous platinum nanoparticles. Small 2006, 2, 249-253.

[46] Tian, N.; Zhou, Z. Y.; Sun, S. G.; Ding, Y.; Wang, Z. L. Synthesis of tetrahexahedral platinum nanocrystals with high-index facets and high electro-oxidation activity. Science 2007, 316, 732-735.

[47] Lim, B.; Lu, X.; Jiang, M.; Camargo, P. H. C.; Cho, E. C.; Lee, E. P.; Xia, Y. Facile synthesis of highly faceted multioctahedral Pt nanocrystals through controlled overgrowth. Nano Lett. 2008, 8, 4043-4047.

[48] Wang, C.; Daimon, H.; Onodera, T.; Koda, T.; Sun, S. A general approach to the size- and shape-controlled synthesis of platinum nanoparticles and their catalytic reduction of oxygen. Angew. Chem. Int. Ed. 2008, 47, 3588-3591.

[49] Schmidt, T. J.; Gasteiger, H. A.; Stäb, G. D.; Urban, P. M.; KoIb, D. M.; Behm, R. J. Characterization of high-surface-area electrocatalysts using a rotating disk electrode configuration. J. Electrochem. Soc. 1998, 145, 2354-2358.

[50] Gasteiger, H. A.; Kocha, S. S.; Sompalli, B.; Wagner, F. T. Activity benchmarks and requirements for Pt, Pt-alloy, and non-Pt oxygen reduction catalysts for PEMFCs. Appl. Catal. $B$ 2005, 56, 9-35.

[51] Stamenkovic, V. R.; Fowler, B.; Mun, B. S.; Wang, G.; Ross, P. N.; Lucas, C. A.; Marković, N. M. Improved oxygen reduction activity on $\mathrm{Pt}_{3} \mathrm{Ni}(111)$ via increased surface site availability. Science 2007, 315, 493-497.

[52] Arenz, M.; Stamenkovic, V.; Schmidt, T. J.; Wandelt, K.; Ross, P. N.; Markovic, N. M. The electro-oxidation of formic acid on Pt-Pd single crystal bimetallic surfaces. Phys. Chem. Chem. Phys. 2003, 5, 4242-4251.

[53] Adzic, R. R.; Tripkovic, A. V; O'Grady, W. E. Structural effects in electrocatalysis. Nature 1982, 296, 137-138.

[54] Lim, B.; Jiang, M.; Tao, J.; Camargo, P. H. C.; Zhu, Y.; Xia, $\mathrm{Y}$. Shape-controlled synthesis of $\mathrm{Pd}$ nanocrystals in aqueous solutions. Adv. Funct. Mater. 2009, 19, 189-200. 